\title{
Similarity solutions for granular flows in hoppers
}

\author{
Pierre-Alain Gremaud*, John V. Matthews $\dagger$ and Michael Shearer $\ddagger$
}

\begin{abstract}
Similarity solutions for the steady state equations governing flow of granular materials under gravity in a conical or wedge-shaped hopper are constructed. Such solutions can be considered for various plasticity models. Results obtained under the classical Mohr-Coulomb yield condition are compared with those obtained when a von Mises yield condition is imposed. A thorough stability study in the case of Mohr-Coulomb materials, for which several simplifications take place, is proposed.
\end{abstract}

AMS(MOS) subject classifications. 65L10, 73E05, 35Q72

\section{INTRODUCTION}

In the early 1960 's, Jenike [7] discovered similarity solutions for the steady state equations governing flow of granular materials under gravity in a conical or wedgeshaped hopper, see Figure 1.1. In these solutions, particle paths are radial lines converging on the vertex of the hopper. For this reason, the solutions are referred to as radial solutions. The similarity is reflected in scalings of the stress and velocity with respect to radial distance $r$, with the result that stress decreases along particle paths while the particles accelerate. Radial solutions, tabulated by Jenike for a large range of physical parameters, form the basis for much work on the design of mass flow hoppers [8], in which the flow is thought to be approximately radial. These solutions are also helpful for understanding and studying basic properties of hopper flow [13], and the role of parameters such as internal friction and wall friction.

In Section 2, we describe the equations of motion in detail, in two and three dimensions. The radial solutions are constructed in Section 3. We compare results under the classical Mohr-Coulomb yield condition, with those obtained when the more sophisticated von Mises condition is imposed. In each case, the stress equations can be solved independently of the velocity when considering radial solutions. However, this decoupling of the stress equations extends to non-radial solutions only in the Mohr-Coulomb case.

For physically realistic ranges of parameters [15], the steady state Mohr-Coulomb equations are hyperbolic with $t=\log r$ being the time-like variable, decreasing as the particles travel down the hopper. (In the von Mises case, the equations are not hyperbolic for all parameter values [15].) In solving the steady-state equations numerically for non-radial solutions, it is common practice $[13,14]$ to use numerical methods that

* Department of Mathematics and Center for Research in Scientific Computing, North Carolina State University, Raleigh, NC 27695-8205, gremaud@math.ncsu.edu. Supported by the Army Research Office (ARO) through grants DAAH04-95-1-0419 and DAAH04-96-1-0097, by the National Science Foundation (NSF) through grant DMS-9818900, and by a grant from the North Carolina Supercomputing Center.

†Department of Mathematics, North Carolina State University, Raleigh, NC 27695-8205, jvmatthe@eos.ncsu.edu. Supported by ARO grant DAAH04-96-1-0097, and by a grant from the North Carolina Supercomputing Center.

$\ddagger$ Department of Mathematics and Center for Research in Scientific Computing, North Carolina State University, Raleigh, NC 27695-8205, shearer@math.ncsu.edu. Supported through ARO grant DAAG55-98-10128 and NSF grant DMS-9818900. 
exploit the hyperbolic structure of the equations, such as the method of characteristics. (Since the equations are nonlinear, it is not surprising that non-radial solutions break down at finite $t$ [13], but that topic will be explored in a future paper.) The main topic of this paper is the linearized stability of the radial solutions to perturbations high in the hopper. More specifically, we investigate how an initial perturbation of the radial solution at $t=t_{0}$ propagates down the hopper (as $t=\log r$ decreases).

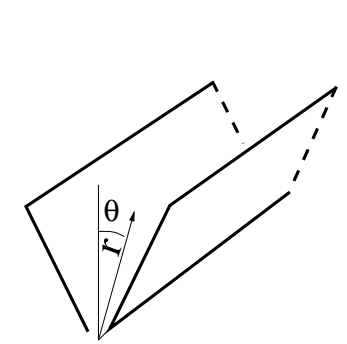

wedge-shaped hopper

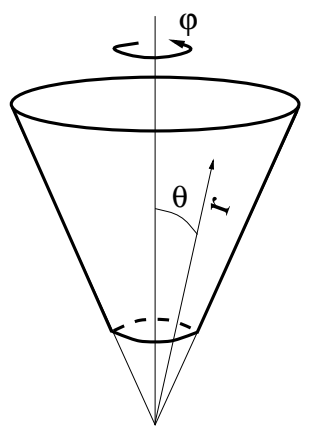

conical hopper

FIGURE 1.1. Geometry and coordinate systems for the two dimensional, wedge-shaped

hopper and the three dimensional conical hopper.

Linearizing the equations about the radial solution gives an eigenvalue problem for the growth rate of the perturbation. For the Mohr-Coulomb yield condition, the eigenvalue problem reflects the decoupling of the stress equations from the velocity equations, thus greatly simplifying the problem. But in the von Mises case, there is no such decoupling, and the eigenvalue problem is large and somewhat intractable. For this reason, our study of linearized stability is restricted to the Mohr-Coulomb yield condition. We later point out how the added complication arises in the von Mises case.

After linearization, we compute the eigenvalues numerically for various values of the parameters, in two and three dimensions. The reliability of the results is checked in various ways. We consider for instance the case of smooth walls, for which a small angle approximation (for sine and cosine of a dependent variable) leads to a much simplified eigenvalue problem that can be solved explicitly in two dimensions, and nearly explicitly in three dimensions. (In three dimensions, after a further small angle approximation, this time in the opening angle of the hopper, the eigenvalue problem reduces to a Bessel equation.) In the smooth wall case, the eigenvalues lie on a vertical line in the complex plane, the location of the line with respect to the origin indicating growth or decay of the perturbation, and hence stability or instability. The computed eigenvalues agree very closely with those of the approximations. The stability study is presented in Section 4.

\section{THE MODELS}

We consider steady incompressible flow in which the particles have no motion in the axial (or $\varphi$ ) direction. Then the dependent variables reduce to four components 
of stress and two of velocity:

$$
T=\left(\begin{array}{ccc}
T_{r r} & T_{r \vartheta} & 0 \\
T_{r \vartheta} & T_{\vartheta \vartheta} & 0 \\
0 & 0 & T_{\varphi \varphi}
\end{array}\right) \quad v=\left(\begin{array}{c}
v_{r} \\
v_{\vartheta} \\
0
\end{array}\right) .
$$

The equations of motion are

$$
\nabla \cdot T=\rho g
$$

and

$$
\nabla \cdot v=0,
$$

in which $\rho$ is the density, taken to be constant, and the vector $g$ is the acceleration due to gravity. Equation (2.3) expresses incompressibility, while equations (2.2) represent the balance of forces in the material. Note that because gravity has no component in the axial direction, $T$ is independent of $\varphi$, and given the form (2.1) of the stress tensor $T$, equations (2.2) reduce to two equations, so that the system $(2.2,2.3)$ consists of three equations in the six unknowns of (2.1).

Plastic deformation is assumed everywhere. Constitutive models based on plasticity are conveniently expressed in terms of the principal stresses $\sigma_{i}, i=1,2,3$, i.e., the eigenvalues of the stress tensor $T$. If the principal stresses are ordered $\sigma_{1} \geq \sigma_{2} \geq \sigma_{3}$, then the Mohr-Coulomb yield condition takes the form

$$
\frac{\sigma_{1}}{\sigma_{3}}=\frac{1+\sin \delta}{1-\sin \delta}
$$

where $\delta$ is the angle of internal friction. This relation can be derived from the law of sliding friction [13, chap. 3]. By introducing an "average" stress $q=\frac{1}{2}\left(\sigma_{1}+\sigma_{3}\right)$, one can easily rewrite the yield condition as

$$
\left(\sigma_{1}-q\right)^{2}+\left(\sigma_{3}-q\right)^{2}=2 q^{2} \sin ^{2} \delta .
$$

Further, (2.4) can be expressed in the original stress variables

$$
\left(T_{r r}-T_{\vartheta \vartheta}\right)^{2}+4 T_{r \vartheta}^{2}=\sin ^{2} \delta\left(T_{r r}+T_{\vartheta \vartheta}\right)^{2} .
$$

An alternative condition, known as the von Mises yield condition, is expressed through the average of the principal stresses $p=\frac{1}{3}\left(\sigma_{1}+\sigma_{2}+\sigma_{3}\right)$. This condition can be written

$$
\left(\sigma_{1}-p\right)^{2}+\left(\sigma_{2}-p\right)^{2}+\left(\sigma_{3}-p\right)^{2}=2 p^{2} \sin ^{2} \delta
$$

or equivalently

$$
\left(T_{r r}-p\right)^{2}+\left(T_{\vartheta \vartheta}-p\right)^{2}+\left(T_{\varphi \varphi}-p\right)^{2}+2 T_{r \vartheta}^{2}=2 \sin ^{2} \delta p^{2} .
$$

The similarity between (2.4) and (2.5) should be noted.

Additional constitutive assumptions are needed to close the systems. In the MohrCoulomb analysis, $\sigma_{2}$ does not appear. The Haar-von Karman assumption can be invoked to evaluate the circumferential stress $T_{\varphi \varphi}$. Indeed, the Mohr-Coulomb analysis merely states $\sigma_{1} \geq T_{\varphi \varphi} \geq \sigma_{3}$. For axisymmetric converging hoppers, the Haar-von Karman assumption states that $T_{\varphi \varphi}$ is in fact the major principal stress, i.e.,

$$
T_{\varphi \varphi}=\sigma_{1}=q(1+\sin \delta) \text {. }
$$


The flow rule, which relates the stress to the deformation through the strain rate tensor, defined as

$$
V=-\frac{1}{2}\left(\nabla v+(\nabla v)^{T}\right)
$$

is taken according to the principle of coaxiality. In other words, the stress tensor $T$ and the strain rate tensor $V$ are assumed to have parallel eigenvectors.

In the von Mises analysis, we adopt Levy's flow rule, which states that the strain rate tensor $V$ is proportional to the deviatoric part of the stress tensor $T-p \mathbb{I}$, i.e.

$$
V=\chi(T-p \mathbb{I})
$$

where $\chi$ is a positive scalar. Note that Levy's flow rule both determines the circumferential stress and imposes coaxility.

In the coordinates of Figure 1.1, the strain rate tensor $V$ takes the form

$$
V=\left(\begin{array}{ccc}
-\partial_{r} v_{r} & -\frac{\partial_{\vartheta} v_{r}}{2 r}-\frac{\partial_{r} v_{\vartheta}}{2}+\frac{v_{\vartheta}}{2 r} & 0 \\
-\frac{\partial_{\vartheta} v_{r}}{2 r}-\frac{\partial_{r} v_{\vartheta}}{2}+\frac{v_{\vartheta}}{2 r} & -\frac{1}{r}\left(\partial_{\vartheta} v_{\vartheta}+v_{r}\right) & 0 \\
0 & 0 & -\frac{v_{r} \sin \vartheta+v_{\vartheta} \cos \vartheta}{r \sin \vartheta}
\end{array}\right)
$$

The equations corresponding to $(2.2,2.3)$ can now be written for both sets of material assumptions discussed above, i.e., Mohr-Coulomb and von Mises analyses.

\section{Equations of motion for Mohr Coulomb flows.}

$$
\begin{gathered}
\partial_{r} T_{r r}+\frac{1}{r} \partial_{\vartheta} T_{r \vartheta}+\frac{2 T_{r r}}{r}+\frac{\cot \vartheta}{r} T_{r \vartheta}-\frac{1}{r}\left(T_{\vartheta \vartheta}+T_{\varphi \varphi}\right)=-\rho g \cos \vartheta \\
\partial_{r} T_{r \vartheta}+\frac{1}{r} \partial_{\vartheta} T_{\vartheta \vartheta}+\frac{3}{r} T_{r \vartheta}+\frac{1}{r} \cot \vartheta\left(T_{\vartheta \vartheta}-T_{\varphi \varphi}\right)=\rho g \sin \vartheta \\
\left(T_{r r}-T_{\vartheta \vartheta}\right)^{2}+4 T_{r \vartheta}^{2}=\sin ^{2} \delta\left(T_{r r}+T_{\vartheta \vartheta}\right)^{2} \\
T_{\varphi \varphi}=\frac{1}{2}\left(T_{r r}+T_{\vartheta \vartheta}\right)(1+\sin \delta) \\
\partial_{r} v_{r}+\frac{1}{r} \partial_{\vartheta} v_{\vartheta}+\frac{2}{r} v_{r}+\frac{1}{r} \cot \vartheta v_{\vartheta}=0 \\
\left(\partial_{r} v_{r}-\frac{\partial_{\vartheta} v_{\vartheta}}{r}-\frac{v_{r}}{r}\right) \frac{2 T_{r \vartheta}}{T_{r r}-T_{\vartheta \vartheta}}-\left(\partial_{r} v_{\vartheta}+\frac{\partial_{\vartheta} v_{r}}{r}-\frac{v_{\vartheta}}{r}\right)=0
\end{gathered}
$$

Equations of Motion For von Mises flows.

$$
\begin{gathered}
\partial_{r} T_{r r}+\frac{1}{r} \partial_{\vartheta} T_{r \vartheta}+\frac{2 T_{r r}}{r}+\frac{\cot \vartheta}{r} T_{r \vartheta}-\frac{1}{r}\left(T_{\vartheta \vartheta}+T_{\varphi \varphi}\right)=-\rho g \cos \vartheta \\
\partial_{r} T_{r \vartheta}+\frac{1}{r} \partial_{\vartheta} T_{\vartheta \vartheta}+\frac{3}{r} T_{r \vartheta}+\frac{1}{r} \cot \vartheta\left(T_{\vartheta \vartheta}-T_{\varphi \varphi}\right)=\rho g \sin \vartheta \\
\left(T_{r r}-p\right)^{2}+\left(T_{\vartheta \vartheta}-p\right)^{2}+\left(T_{\varphi \varphi}-p\right)^{2}+2 T_{r \vartheta}^{2}=2 \sin ^{2} \delta p^{2}, \\
\frac{v_{r}+\cot \vartheta v_{\vartheta}}{\partial_{\vartheta} v_{\vartheta}+v_{r}}=\frac{T_{\varphi \varphi}-p}{T_{\vartheta \vartheta}-p},
\end{gathered}
$$




$$
\begin{gathered}
\partial_{r} v_{r}+\frac{1}{r} \partial_{\vartheta} v_{\vartheta}+\frac{2}{r} v_{r}+\frac{1}{r} \cot \vartheta v_{\vartheta}=0 \\
\left(\frac{1}{r} \partial_{\vartheta} v_{\vartheta}+\frac{1}{r} v_{r}\right) \frac{2 T_{r \vartheta}}{T_{\vartheta \vartheta}-p}-\left(\partial_{r} v_{\vartheta}+\frac{1}{r} \partial_{\vartheta} v_{r}-\frac{1}{r} v_{\vartheta}\right)=0 .
\end{gathered}
$$

Note that in the Mohr-Coulomb analysis, the stress equations are decoupled from the velocity equations. In the von Mises analysis, the system is fully coupled.

We conclude this section with the description of suitable boundary conditions. For the stress variables, the boundary conditions are given by the law of sliding friction. At any point on the wall, the magnitude of the tangential stress $\left|T_{T}\right|$ is proportional to the magnitude of the normal stress $\left|T_{N}\right|$, i.e.,

$$
\left|T_{T}\right|=\mu\left|T_{N}\right|, \quad \text { on } \Gamma,
$$

where $\mu>0$ is the coefficient of wall friction. If $N$ is the unit outer normal vector to $\Gamma$, then

$$
T_{N}=T_{i j} N_{i} N_{j}, \quad\left(T_{T}\right)_{i}=T_{i j} N_{j}-T_{N} N_{i} .
$$

In our coordinate system, $\Omega$ being axisymmetric, we have $N=\left(\begin{array}{ll}n_{r}, & n_{\vartheta}, \quad 0\end{array}\right)^{T}$. Fur-

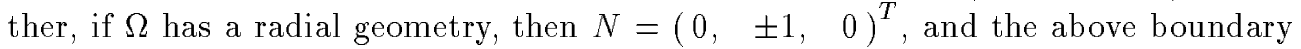
condition becomes

$$
T_{r \vartheta}= \pm \mu T_{\vartheta \vartheta} \quad \text { on } \Gamma
$$

with a "+" sign on one side of the hopper and a "-" sign on the other. The boundary condition on the velocity is simply

$$
v \cdot N=0, \quad \text { on } \Gamma \text {. }
$$

\section{Radial SOLUtions}

Jenike's radial solutions [7] are similarity solutions for the steady state equations, in an infinite conical hopper $\Omega$, i.e., in spherical coordinates

$$
\Omega=\left\{(r, \vartheta, \varphi) ; r>0,-\vartheta_{w}<\vartheta<\vartheta_{w}, 0<\varphi<\pi\right\} .
$$

This concept of radial solution is widely used in the design of industrial hoppers [8]. Following Jenike, we seek solutions of the equations in the form

$$
T_{i j}(r, \vartheta)=r T_{i j}(\vartheta), \quad v(r, \vartheta)=-\frac{1}{r^{2}} u(\vartheta) e_{r},
$$

where $e_{r}$ is the unit vector in the radial direction.

The formulation can be simplified by working with appropriate representations of the solutions. More precisely, it is possible to "solve" the algebraic constraints (2.4') and (2.5'), and express the stress tensor $T$ in terms of two new scalar variables, usually referred to as Sokolovskii variables. The approach is slightly different in the Mohr-Coulomb and von Mises analyses.

For a Mohr-Coulomb material, we set

$$
\begin{array}{llrl}
T_{r r} & =\sigma(1-\sin \delta \cos 2 \psi), & & T_{\vartheta \vartheta}=\sigma(1+\sin \delta \cos 2 \psi), \\
T_{r \vartheta}=-\sigma \sin \delta \sin 2 \psi, & & T_{\varphi \varphi}=\sigma(1+\sin \delta) .
\end{array}
$$

Note that $\left(2.4^{\prime}\right)$ is then automatically verified. 
For a von Mises material, the flow rule (2.7) implies

$$
\frac{T_{\vartheta \vartheta}-p}{T_{\varphi \varphi}-p}=\frac{V_{\vartheta \vartheta}}{V_{\varphi \varphi}}=1
$$

where the last equality is a direct consequence of the radial character of the velocity. It follows that $T_{\vartheta \vartheta}=T_{\varphi \varphi}$. Taking then into account the yield condition (2.5') and the fact that due to the axial symmetry, $e_{\varphi}$ must be an eigenvector of $T$, the following representation of $T$ can be considered

$$
\begin{aligned}
T_{r r} & =\sigma\left(1-\frac{2}{\sqrt{3}} \sin \delta \cos 2 \psi\right), & & T_{\vartheta \vartheta}=\sigma\left(1+\frac{1}{\sqrt{3}} \sin \delta \cos 2 \psi\right), \\
T_{r \vartheta} & =-\sigma \sin \delta \sin 2 \psi, & T_{\varphi \varphi} & =\sigma\left(1+\frac{1}{\sqrt{3}} \sin \delta \cos 2 \psi\right),
\end{aligned}
$$

where $\sigma=p$ is the average stress. The substitutions $\sigma(r, \vartheta)=r \sigma(\vartheta)$ and $\psi(r, \vartheta)=$ $\psi(\vartheta)$ lead to systems of ODEs. Note that in the context of radial solutions, the stress and velocity equations are decoupled for both Mohr-Coulomb and von Mises models.

Considering first the stress equations, we get the following type of boundary value problem

$$
\begin{aligned}
\mathbb{A}\left(\begin{array}{c}
\sigma^{\prime}(\vartheta) \\
\psi^{\prime}(\vartheta)
\end{array}\right) & =\mathbb{F}(\vartheta, \sigma(\vartheta), \psi(\vartheta)), \quad \vartheta \in\left(-\vartheta_{w}, \vartheta_{w}\right), \\
\psi\left( \pm \vartheta_{w}\right) & = \pm \psi_{w},
\end{aligned}
$$

where the quantities $\mathbb{A}, \mathbb{F}$ and $\psi_{w}$ depend on the model considered. In the MohrCoulomb case, we have

$$
\mathbb{A}=\left(\begin{array}{cc}
-\sin \delta \sin 2 \psi & -2 \sin \delta \sigma \cos 2 \psi \\
1+\sin \delta \cos 2 \psi & -2 \sin \delta \sigma \sin 2 \psi
\end{array}\right) \quad \text { and } \psi_{w}=\frac{1}{2}\left(\Phi+\arcsin \left(\frac{\sin \Phi}{\sin \delta}\right)\right)
$$

where $\Phi$ is the angle of wall friction, which is defined by $\tan \Phi=\mu, 0 \leq \Phi \leq \pi / 2$. The corresponding expressions in the von Mises case are

$\mathbb{A}=\left(\begin{array}{cc}-\sin \delta \sin 2 \psi & -2 \sin \delta \sigma \cos 2 \psi \\ 1+\frac{1}{\sqrt{3}} \sin \delta \cos 2 \psi & -\frac{2}{\sqrt{3}} \sin \delta \sigma \sin 2 \psi\end{array}\right), \psi_{w}=\frac{1}{2}\left(\Phi+\arcsin \left(\sqrt{3} \frac{\sin \Phi}{\sin \delta}\right)\right)$,

in which $\tan \Phi=\mu / \sqrt{3}$. We omit the expressions for $\mathbb{F}$ in both cases as they are easily found.

The two systems of ODEs for the stresses have similar, but not identical properties. For instance, in the Mohr-Coulomb analysis, the stress equations are nonsingular, i.e., the matrix $\mathbb{A}$ is nonsingular, provided that the angular Sokolovskii variable $\psi$ satisfies

$$
|\psi|<\frac{\pi}{4}+\frac{\delta}{2}
$$

while in the von Mises case, the corresponding, and more stringent, condition is

$$
|\psi|<\frac{\pi}{2}-\frac{1}{2} \arccos \left(\frac{\sin \delta}{\sqrt{3}}\right) \leq \frac{\pi}{4}+\frac{\delta}{2} .
$$

For both models, the substitution $v(r, \vartheta)=-\frac{1}{r^{2}} u(\vartheta) e_{r}$, where $u$ is a scalar function, reduces the velocity equations to a single ODE. For a Mohr-Coulomb material, 
this equation is found to be

$$
u^{\prime}(\vartheta)=-3 u(\vartheta) \tan 2 \psi(\vartheta), \quad \vartheta \in\left(-\vartheta_{w}, \vartheta_{w}\right) .
$$

In the von Mises case, the equation is

$$
u^{\prime}(\vartheta)=-\sqrt{12} u(\vartheta) \tan 2 \psi(\vartheta), \quad \vartheta \in\left(-\vartheta_{w}, \vartheta_{w}\right) .
$$
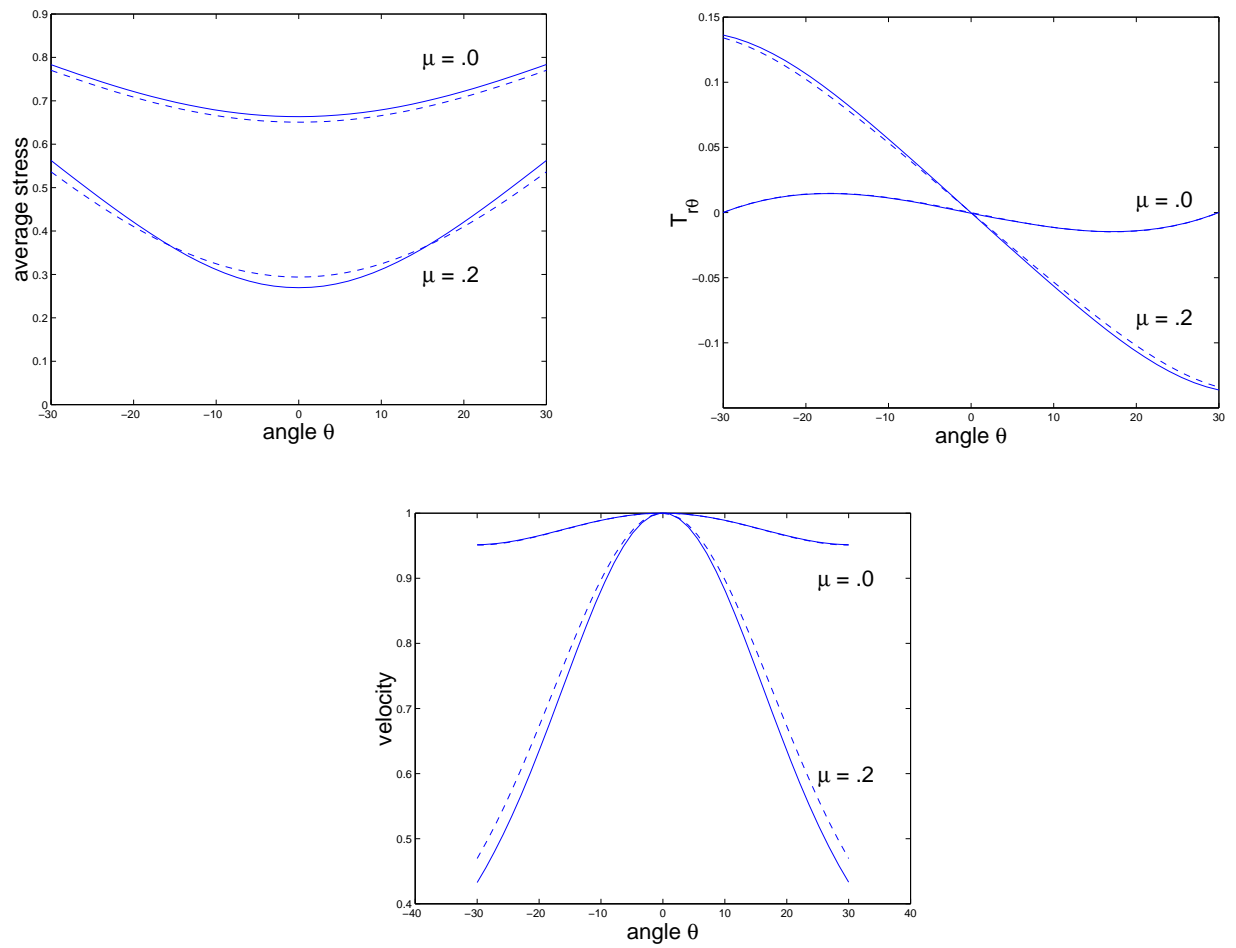

Figure 3.1. Comparison of the values of the stress and velocity fields between MohrCoulomb, solid lines, and von Mises, dashed lines; angle of friction $\delta=30^{\circ}$, half opening angle $\vartheta_{w}=30^{\circ}$; wall friction $\mu=0$ and $\mu=.2$.

In both cases, $\psi$ is obtained from (3.3). No boundary conditions are imposed on $u$. Instead, the velocity fields are normalized by imposing the condition $u(0)=1$. An alternative would be to specify the withdrawal rate $\rho \int v \cdot e_{r} r d \vartheta$.

Differences between the two models are illustrated for various values of wall friction $\mu$ in Figures 3.1 and 3.2. Figure 3.1 shows that for moderate roughness of the wall, both approaches yield comparable results. If the roughness of the walls is large however, as illustrated in Figure 3.2, the difference between the predictions of the two models becomes large. This is especially true of the velocity fields.

Figure 3.2, left, shows that while for this configuration the Mohr-Coulomb analysis predicts an essentially stagnant part of the flow, for $20^{\circ} \lesssim|\vartheta| \leq 30^{\circ}$, no such phenomenon occurs for the von Mises analysis. Note that this phenomenon corresponds to the velocity equation becoming singular. Indeed, both of the velocity equations $(3.4,3.5)$ become singular when $\psi$ crosses $\pi / 4$. It can be observed in Figure 3.2, right, 
that $\psi$ is quite close to $\pi / 4 \approx .7854$ at a location roughly corresponding to the edge of the "stagnant zone".
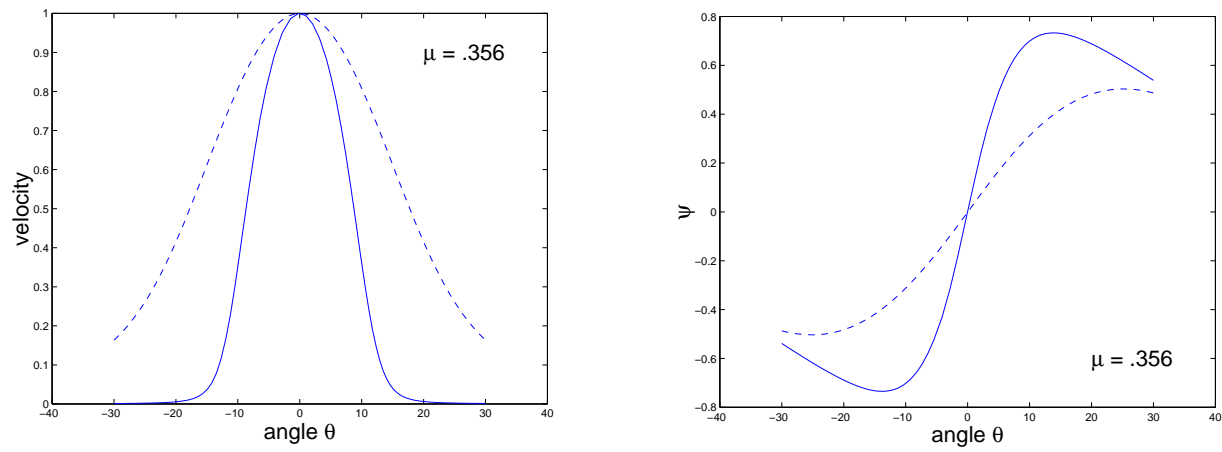

FIGURE 3.2. Comparison of the values of the velocity and angular variable $\psi$ between Mohr-Coulomb, solid lines, and von Mises, dashed lines; angle of friction $\delta=30^{\circ}$, half opening angle $\vartheta_{w}=30^{\circ}$; wall friction $\mu=.356$.

\section{Stability}

The goal of this section is to analyze the stability of the Mohr-Coulomb similarity solutions introduced in the previous section. We focus exclusively on the cases of Mohr Coulomb materials for two reasons. First, as suggested by the results of the previous section, the Mohr-Coulomb approach predicts stagnant zones for some parameter values for which the von Mises approach predicts none (see also [13]). Thus the Mohr-Coulomb approach should lead to more conservative estimates when used for design purposes. Second, the analysis can be done by focusing exclusively on the stress equations, which decouple from the velocity equations. A similar study for von Mises materials for which there is no such decoupling is thus far more challenging, and will not be presented here. More specifically, the Haar von Karman hypothesis associated with Mohr-Coulomb materials is embodied in equation (2.6'). The corresponding equation for von Mises material is equation (2.13), which collapses to a triviality for radial solutions, but after linearization couples stresses to velocities in an essential way.

For the sake of completeness, the case of two dimensional flows, for which the curvature of the hopper wall is neglected (wedge shaped hopper) is also included. The present results complement those of [14]. We focus exclusively on the stress equations, which we linearize about the radial stress field. More precisely, we rewrite the Sokolovskii variables as

$$
\begin{aligned}
& \sigma(r, \vartheta)=r\left(\sigma^{0}(\vartheta)+\varepsilon \sigma^{1}(r, \vartheta)\right) \\
& \psi(r, \vartheta)=\psi^{0}(\vartheta)+\varepsilon \psi^{1}(r, \vartheta)
\end{aligned}
$$

where $\sigma^{0}$ and $\psi^{0}$ correspond to the radial solutions of $\S 3$ and $\sigma^{1}$ and $\psi^{1}$ are corresponding perturbations, $\varepsilon$ being small and positive. After substituting the above expressions into $(2.8,2.9)$, we obtain

$$
\left(\begin{array}{l}
\sigma^{1} \\
\psi^{1}
\end{array}\right)_{t}+\mathbb{A}\left(\begin{array}{l}
\sigma^{1} \\
\psi^{1}
\end{array}\right)_{\vartheta}+\mathbb{B}\left(\begin{array}{c}
\sigma^{1} \\
\psi^{1}
\end{array}\right)+(d-2) \mathbb{C}\left(\begin{array}{l}
\sigma^{1} \\
\psi^{1}
\end{array}\right)=0
$$


where $d$ is the dimension $(d=2$ or 3$), t=\log r$ and

$$
\begin{aligned}
\mathbb{A} & =\frac{1}{2 \sigma^{0} s\left(s-\cos \left(2 \psi^{0}\right)\right)}\left(\begin{array}{cc}
-2 s \sigma^{0} \sin \left(2 \psi^{0}\right) & 8 s^{2}\left(\sigma^{0}\right)^{2} \\
\left(1-s^{2}\right) / 2 & -2 s \sigma^{0} \sin \left(2 \psi^{0}\right)
\end{array}\right), \\
\mathbb{B} & =\frac{1}{2 \sigma^{0} s\left(s-\cos \left(2 \psi^{0}\right)\right)} \\
& \left(\begin{array}{cc}
2 \sigma^{0} s^{2}\left(3+2\left(\psi^{0}\right)^{\prime}\right)-2 \sigma^{0} s \cos \left(2 \psi^{0}\right) & 8 s^{2} \sigma^{0}\left(\sigma^{0}\right)^{\prime} \\
-s \sin \left(2 \psi^{0}\right)\left(1+\left(\psi^{0}\right)^{\prime}\right) & 2 s \sigma^{0}\left(s-\cos \left(2 \psi^{0}\right)\right)\left(3+2\left(\psi^{0}\right)^{\prime}\right)-2 s\left(\sigma^{0}\right)^{\prime} \sin \left(2 \psi^{0}\right)
\end{array}\right), \\
\mathbb{C}=\frac{s}{2 \sigma^{0} s\left(s-\cos \left(2 \psi^{0}\right)\right)} & 8\left(\begin{array}{cc}
2 \sigma_{0} s\left(1+\cos \left(2 \psi^{0}\right)+\sin \left(2 \psi^{0}\right) \cot \vartheta\right) & \sigma^{2} s \cot \vartheta \\
-(1+s)\left(\sin \left(2 \psi^{0}\right)+\left(1-\cos \left(2 \psi^{0}\right)\right) \cot \vartheta\right) / 2 & 2 \sigma^{0}\left(s-\cos \left(2 \psi^{0}\right)-\sin \left(2 \psi^{0}\right) \cot \vartheta\right)
\end{array}\right) .
\end{aligned}
$$

Here, we have set $s=\sin \delta$. We separate the variables by setting

$$
\sigma^{1}(t, \vartheta)=\Sigma(\vartheta) e^{-\lambda t}, \quad \psi^{1}(t, \vartheta)=\Psi(\vartheta) e^{-\lambda t} .
$$

Since $t=\log r$ tends to $-\infty$ when $r \rightarrow 0$, stability corresponds to eigenvalues with negative real parts. From (4.1), we derive a discretized eigenvalue problem

$$
(\mathbb{A D}+\mathbb{B}+(d-2) \mathbb{C})\left(\begin{array}{l}
\Sigma \\
\Psi
\end{array}\right)=\lambda\left(\begin{array}{l}
\Sigma \\
\Psi
\end{array}\right)
$$

where

$$
\mathbb{D}=\left(\begin{array}{cc}
\mathbb{D}_{\Sigma} & 0 \\
0 & \mathbb{D}_{\Psi}
\end{array}\right)
$$

in which $\mathbb{D}_{\Sigma}$ is a discretization of $\frac{d}{d \vartheta}$ and $\mathbb{D}_{\Psi}$ is a discretization of $\frac{d}{d \vartheta}$ with homogeneous Dirichlet boundary conditions. The matrix $\mathbb{D}$ is constructed by a pseudospectral Chebyshev collocation method $\left[4\right.$, p.65-70]. To obtain $\mathbb{D}_{\Psi}$, we simply "collocate" $\Psi$ at $\pm \theta_{w}$, in effect constructing $\mathbb{D}_{\Psi}$ from $\mathbb{D}_{\Sigma}$ by removing the first and last rows and the first and last columns.

For comparison purposes, we note that if some further simplifications are considered, it is possible in some cases to find the exact spectrum. A "smooth wall analysis" is such a case. First, we make the small angle approximation in $\psi$, then we consider smooth walls, for which $\mu=0$, simplifying the boundary condition (2.15). Following [11] and [14], $\psi$ in (3.3) is assumed to be small in absolute value. Then the stress components are simplified by using $\sin (2 \psi) \approx 2 \psi$ and $\cos (2 \psi) \approx 1$. If, for convenience, the new variable $\omega=\sigma \psi$ is introduced, the perturbation equations corresponding to (4.1) become

$$
\left(\begin{array}{c}
\sigma^{1} \\
\omega^{1}
\end{array}\right)_{t}+\tilde{\mathbb{A}}\left(\begin{array}{c}
\sigma^{1} \\
\omega^{1}
\end{array}\right)_{\vartheta}+\tilde{\mathbb{B}}\left(\begin{array}{c}
\sigma^{1} \\
\omega^{1}
\end{array}\right)+(d-2) \tilde{\mathbb{C}}\left(\begin{array}{c}
\sigma^{1} \\
\omega^{1}
\end{array}\right)=0
$$

with

$$
\tilde{\mathbb{A}}=\left(\begin{array}{cc}
0 & -\frac{2 s}{1-s} \\
-\frac{1+s}{2 s} & 0
\end{array}\right), \quad \tilde{\mathbb{B}}=\left(\begin{array}{cc}
\frac{1-3 s}{1-s} & 0 \\
0 & 3
\end{array}\right), \quad \tilde{\mathbb{C}}=\left(\begin{array}{cc}
-\frac{2 s}{1-s} & -\frac{2 s \cot \vartheta}{1-s} \\
0 & 1
\end{array}\right) .
$$

Separating again the variables

$$
\sigma^{1}(t, \vartheta)=\tilde{\Sigma}(\vartheta) e^{-\tilde{\lambda} t}, \quad \omega^{1}(t, \vartheta)=\tilde{\Omega}(\vartheta) e^{-\tilde{\lambda} t},
$$


it is here possible to combine the equivalent of (4.2) into a single second order equation in one variable, thanks to the structure of $\tilde{\mathbb{A}}$. One finds

$$
(1+s) \tilde{\Omega}^{\prime \prime}-\left((1-s) \tilde{\lambda}^{2}+(-4+6 s) \tilde{\lambda}+3(1-3 s)\right) \tilde{\Omega}=0
$$

in the two dimensional case and

$$
(1+s) \tilde{\Omega}^{\prime \prime}+(1+s)(\tilde{\Omega} \cot \vartheta)^{\prime}-\left((1-s) \tilde{\lambda}^{2}+(-5+9 s) \tilde{\lambda}+4-20 s\right) \tilde{\Omega}=0
$$

in the three dimensional case. (This corrects a minor error in the corresponding equation (3.24) of [14].)

In the smooth wall case, the ordinary differential equation is supplemented by the boundary condition

$$
\tilde{\Omega}\left( \pm \vartheta_{w}\right)=0
$$

The analysis of the two-dimensional problem $(4.4,4.6)$ is easy. The eigenfunctions are given by

$$
\left\{\sin \left(n \pi \frac{\theta}{\theta_{w}}\right)\right\}_{n=1,2, \ldots} \quad \text { and } \quad\left\{\cos \left(\left(n+\frac{1}{2}\right) \frac{\pi \theta}{\theta_{w}}\right)\right\}_{n=0,1,2, \ldots}
$$

provided that, respectively

$$
\begin{aligned}
& \tilde{\lambda}_{n}=\frac{2-3 s}{1-s} \pm \frac{1}{1-s} \sqrt{1-\left(1-s^{2}\right)\left(\frac{n \pi}{\theta_{w}}\right)^{2}}, \quad n=1,2, \\
& \text { or } \quad \tilde{\lambda}_{n}=\frac{2-3 s}{1-s} \pm \frac{1}{1-s} \sqrt{1-\left(1-s^{2}\right)\left(\frac{(2 n+1) \pi}{2 \theta_{w}}\right)^{2}}, \quad n=0,1,2, \ldots
\end{aligned}
$$

All the eigenvalues are defined by pairs on a vertical line through $\frac{2-3 s}{1-s}$ in the complex plane. The value $\frac{2-3 s}{1-s}=0$, i.e., $\delta \approx 41.81^{\circ}$ corresponds to neutral stability. For values of $\delta$ below that threshold, the two dimensional smooth wall problem is predicted to be unstable. One should note however that by symmetry, physically meaningful functions $\sigma$ and $\psi$, which define the radial solution, have to be respectively even and odd. Consequently, $\omega=\sigma \psi$ should be odd and thus so should $\tilde{\Omega}$ in (4.4) and (4.5). Therefore, only the sine eigenfunctions are physically relevant.

The three dimensional equation (4.5) (which has non-constant coefficients) cannot be solved exactly, in contrast to the two-dimensional case (4.4). Nevertheless, one can find an approximate value for neutral stability. Approximating $\cot \vartheta \approx 1 / \vartheta$, we find the equation

$$
\vartheta^{2} \tilde{\Omega}^{\prime \prime}+\vartheta \tilde{\Omega}^{\prime}+\left(\mu \vartheta^{2}-1\right) \tilde{\Omega}=0
$$

where $\mu=-\left(\frac{1-s}{1+s} \tilde{\lambda}^{2}+\frac{-5+9 s}{1+s} \tilde{\lambda}+\frac{4-20 s}{1+s}\right)$. Replacing $\vartheta$ by $\sqrt{\mu} \vartheta,(4.8)$ becomes Bessel's equation of order one. The corresponding eigenfunctions are given by

$$
\left\{J_{1}\left(\sqrt{\mu_{n}} \vartheta\right)\right\}_{n=1,2, \ldots} \quad \text { and } \quad\left\{Y_{1}\left(\sqrt{\mu_{n}} \vartheta\right)\right\}_{n=1,2, \ldots}
$$


where $J_{1}$ and $Y_{1}$ are respectively the Bessel's functions of the first and second kind of index 1 , with the convention $J_{1}(-x)=-J_{1}(x)$ and $Y_{1}(-x)=-Y_{1}(x)$, and $\mu=\mu_{n}$ is defined below from the boundary condition. As was the case for the two dimensional problem, only half of the above functions are physically relevant. Indeed, the singular character of (4.8) at $\vartheta=0$ together with the requirement that $\tilde{\Omega}$ be odd, forces the eigenfunctions to vanish at zero. The function $Y_{1}$ being singular at zero, only the $J_{1^{-}}$ family, which vanishes at zero, contributes to the spectrum. The boundary condition implies that $\mu$ be such that $J_{1}\left(\sqrt{\mu} \vartheta_{w}\right)=0$. Consequently, there is a sequence of admissible $\mu_{n}$

$$
\mu_{n}=\left(\frac{x_{n}}{\vartheta_{w}}\right)^{2}, \quad n=1,2, \ldots,
$$

where $x_{n}$ is the $n$-th positive root of $J_{1}$. The corresponding eigenvalues $\tilde{\lambda}_{n}$ are then determined by

$$
\frac{1-s}{1+s} \tilde{\lambda}_{n}^{2}+\frac{-5+9 s}{1+s} \tilde{\lambda}_{n}+\frac{4-20 s}{1+s}+\mu_{n}=0 .
$$

The discriminant of (4.9) is

$$
\Delta_{n}=\frac{1}{(1+s)^{2}}\left(\left(1+4 \mu_{n}\right) s^{2}+6 s+9-4 \mu_{n}\right), \quad n=1,2, \ldots
$$

This expression has zeros at

$$
s_{n}=\frac{-3 \pm 4 \sqrt{\mu_{n}^{2}-2 \mu_{n}}}{1+4 \mu_{n}}=\frac{-3 \vartheta_{w}^{2} \pm 4 \sqrt{x_{n}^{4}-2 x_{n}^{2} \vartheta_{w}^{2}}}{\vartheta_{w}^{2}+4 x_{n}^{2}} .
$$

Therefore, $\Delta_{n}$ has two distinct zeros provided that $\mu_{n}=\left(\frac{x_{n}}{\vartheta_{w}}\right)^{2}>2$. Taking into account the numerical value of $x_{1}$, we have $\mu_{1}>2$ for any $\vartheta_{w}<2.709 \approx 155^{\circ}$, which is of course always satisfied. We conclude by noticing that the $\Delta_{n}$ 's are in fact all negative unless $\delta$ is excessively large. Indeed for the $\Delta_{n}$ 's to be positive, we should have $s>s_{n}, n=1,2, \ldots$ This is true if and only if

$$
s>\frac{-3 \vartheta_{w}^{2} \pm 4 \sqrt{x_{1}^{4}-2 x_{1}^{2} \vartheta_{w}^{2}}}{\vartheta_{w}^{2}+4 x_{1}^{2}} \equiv f\left(\vartheta_{w}\right) .
$$

Since the above function $f$ is monotonically decreasing on the interval $(0, \pi / 4)$ of interest, and $f(0)=1, f(\pi / 4) \approx .9159$, it follows that unless $\delta>\arcsin (f(\pi / 4)) \approx$ $66.34^{\circ}$ (which does not correspond to realistic values), the discriminants $\Delta_{n}$ are all negative. Consequently, all the eigenvalues $\tilde{\lambda}_{n}$ of (4.9) are complex and satisfy

$$
\Re\left(\tilde{\lambda}_{n}\right)=\frac{1}{2} \frac{-9 s^{2}-4 s+5}{1-s^{2}}, \quad n=1,2, \ldots
$$

The relation $-9 s^{2}-4 s+5=0$ is the neutral stability condition and thus the three dimensional stress equations under the smooth wall assumption are stable provided that $\delta>33.75^{\circ}$.

Therefore, in both the two and three dimensional cases, the eigenvalues, under the smooth wall assumption, are lined up on a vertical line in the complex plane. The dependency of the abscissa of that line is illustrated in Figure 4.1. 
It is observed that for small values of $\delta, \delta<19.47^{0}$, both problems are unstable, the three dimensional problem being the more unstable. For large values of $\delta, \delta>$ $41.81^{\circ}$, both problems are stable, the three dimensional problem being the more stable.

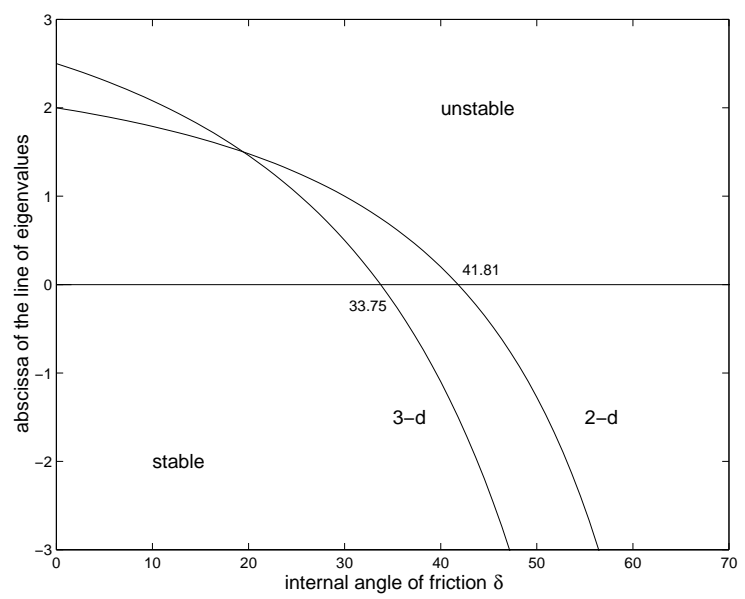

FIGURE 4.1. Smooth wall analysis: comparison of the stability of the two and three dimensional problems.

Incidentally, if in (4.5), the higher order approximation $\cot \vartheta \approx \frac{1}{\vartheta}-\frac{\vartheta}{3}$ is taken, the equation corresponding to (4.8) is

$$
\vartheta^{2} \tilde{\Omega}^{\prime \prime}+\left(\vartheta-\frac{1}{3} \vartheta^{3}\right) \tilde{\Omega}^{\prime}+\left(\left(\mu-\frac{1}{3}\right) \vartheta^{2}-1\right) \tilde{\Omega}=0,
$$

with Dirichlet boundary conditions at $\vartheta=0$ and $\vartheta=\vartheta_{w}$. The equation (4.10) can still be solved exactly by the Frobenius method. One finds

$$
\tilde{\Omega}(\vartheta)=\vartheta \sum_{m=0}^{\infty} \frac{(-2 / 3)^{m} \Gamma\left(\frac{3 \mu}{2}\right) \vartheta^{2 m}}{2^{2 m} \Gamma\left(\frac{3 \mu}{2}-m\right) m !(m+1) !} .
$$

This turns out to be equal to

$$
\tilde{\Omega}(\vartheta)=\vartheta \Phi\left(-\frac{3}{2} \mu+1,2, \frac{1}{6} \vartheta^{2}\right),
$$

where $\Phi$ is the confluent hypergeometric function, i.e.,

$$
\Phi(\alpha, \gamma, z)=1+\frac{\alpha}{\gamma} z+\frac{\alpha(\alpha+1)}{\gamma(\gamma+1)} \frac{z^{2}}{2 !}+\frac{\alpha(\alpha+1)(\alpha+2)}{\gamma(\gamma+1)(\gamma+2)} \frac{z^{3}}{3 !}+\ldots
$$

Easy calculations show that, for $\vartheta_{w}=10^{\circ}$ for instance, the eigenvalues are virtually unchanged, justifying the above assumption $\cot \vartheta \approx 1 / \vartheta$ for small opening angles for the present problem.

The relationship between the eigenvalues $\left\{\tilde{\lambda}_{n}\right\}$ from the smooth wall analysis and the eigenvalues of (4.2) is illustrated in Figure 4.2 in the case $\vartheta_{w}=10^{\circ}, \delta=35^{\circ}$ and $\mu=0$, both in the two and three dimensional cases. 
Based on the above analysis, this value of $\delta=35^{\circ}$ corresponds to an unstable two dimensional problem, and a stable three dimensional one. This is confirmed by Figure 4.2. The displayed eigenvalues have "converged" in the sense that they are not numerical artifacts and do not depend on the size of the mesh (400 nodes). An eigenvalue is kept only if its relative distance the corresponding eigenvalue on a mesh twice as coarse (200 nodes) is less than .001.
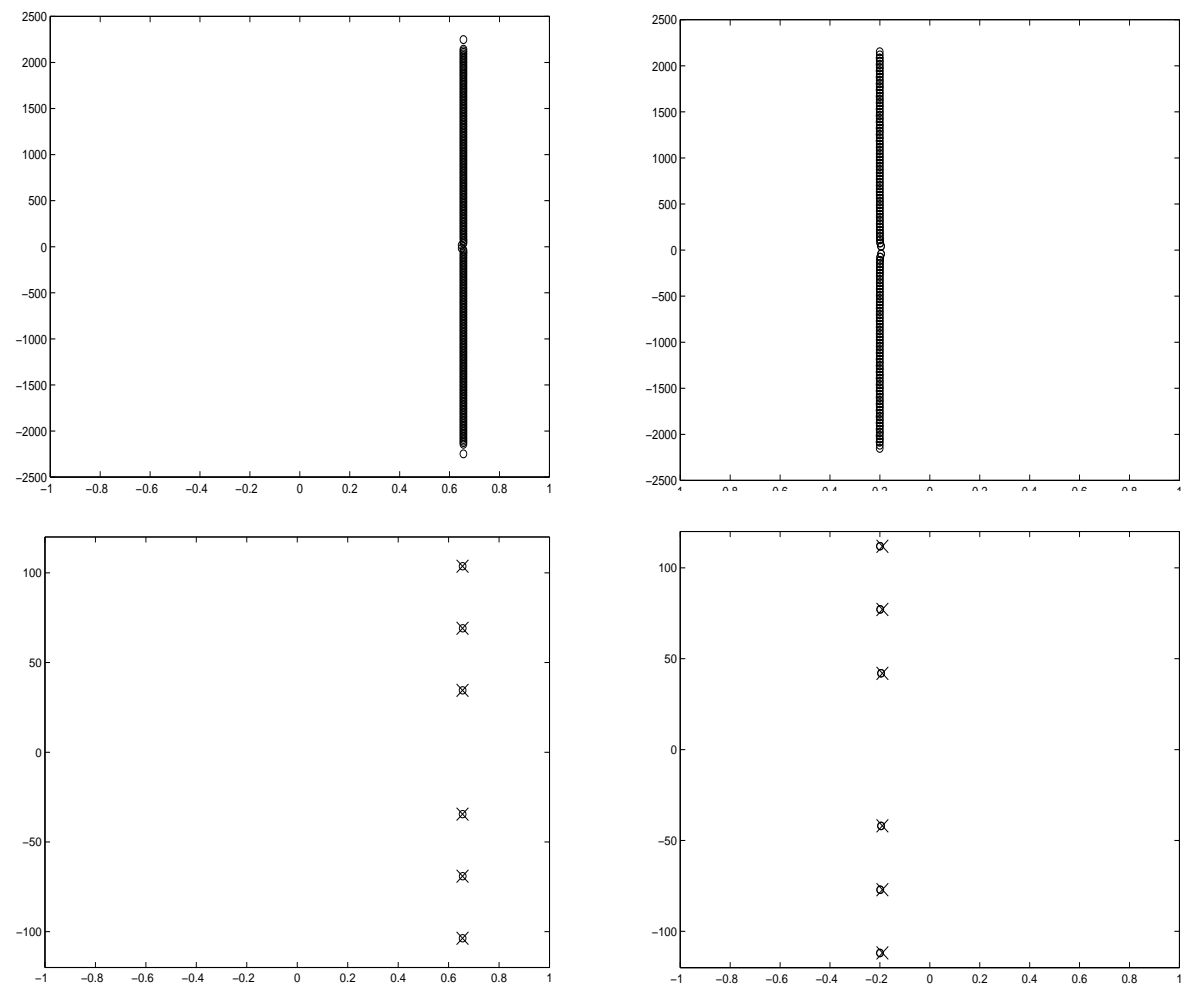

FIGURE 4.2. Discrete spectrum for (4.2) obtained through the Chebyshev collocation method with 400 nodes ( $\operatorname{size}(\mathbb{M})=798$ ). Left column: two dimensional case; right column: three dimensional case. Top: "converged" part of the numerical spectrum (see text below) corresponding to about the first 250 eigenvalues sorted by increasing moduli; bottom left: comparison with the exact eigenvalues of (4.7): $\times$; bottom right; comparison with the exact eigenvalues of $(4.9), \times ; \vartheta_{w}=10^{\circ}, \delta=35^{\circ}, \mu=0$.

As is well known, the eigenvalues of the Chebyshev differentiation matrices for first order derivatives are extraordinarily sensitive to round-off errors [17]. This is related to the fact that scalar first order differentiation operators with boundary conditions have no eigenvalues, and therefore the computed eigenvalues of the corresponding matrices are a pure numerical artifact. Here, the situation is different. Our system behaves more in that respect like a second order operator (wave equation). In fact, the eigenvalues of the matrix $\mathbb{M}=\mathbb{A D}+\mathbb{B}+(d-2) \mathbb{C}$, which corresponds to the right hand side of (4.2) are only mildly sensitive to perturbations. This has been established through a numerical study of the pseudospectrum [16] of $\mathbb{M}$. The corresponding results 
are not reproduced here.

Following [16], further information about the dynamical behavior can be derived from the matrix $\mathbb{M}$. Indeed, if (4.1) is rewritten

$$
\left(\begin{array}{l}
\Sigma \\
\Psi
\end{array}\right)^{\prime}=-\mathbb{M}\left(\begin{array}{c}
\Sigma \\
\Psi
\end{array}\right), \quad t<0
$$

it can be solved as

$$
\left(\begin{array}{l}
\Sigma(t) \\
\Psi(t)
\end{array}\right)=\exp (-t \mathbb{M})\left(\begin{array}{c}
\Sigma(0) \\
\Psi(0)
\end{array}\right)
$$

A plot of $t \mapsto\|\exp (-t \mathbb{M})\|$ gives a first picture of the dynamics of the underlying problems. More precisely, let $\alpha(\mathbb{M})$ be the spectral abscissa of $\mathbb{M}$, i.e.

$$
\alpha(\mathbb{M})=\sup _{z \in \Lambda(\mathbb{M})} \Re(z), \quad \text { with } \Lambda(\mathbb{M})=\text { spectrum of } \mathbb{M} .
$$

Then, if $\mathbb{M}=\mathbb{V} \Lambda \mathbb{V}^{-1}$ is an eigenvalue decomposition of $\mathbb{M}$, one has for any $t<0$

$$
e^{-t \alpha(\mathbb{M})} \leq\|\exp (-t \mathbb{M})\| \leq \kappa(\mathbb{V}) e^{-t \alpha(\mathbb{M})},
$$

where $\kappa(\mathbb{V})=\|\mathbb{V}\|\left\|\mathbb{V}^{-1}\right\|$ is the condition number of the eigenvector matrix $\mathbb{V}$. Note that if $\mathbb{M}$ were normal, which is not the case here, the inequalities in (4.11) would be replaced by equalities. Now, in the case studied so far, i.e., that of Figure 4.2, it is clear that the vertical alignment of the eigenvalues along a line corresponding roughly to $\alpha(\mathbb{M})$ generates sustained oscillations both in the stable (3d) and unstable (2d) case, including in the asymptotic regime $t \rightarrow-\infty$. This behavior is illustrated in Figure 4.3.

All calculations were performed on the "converged part of the spectrum" only, as defined above. For comparison purposes, the lower and upper bounds $e^{-t \alpha(\mathbb{M})}$ and $\kappa(\mathbb{V}) e^{-t \alpha(\mathbb{M})}$ from (4.11) are also displayed in each case. One concludes from Figure 4.3 that the behavior in time of the linearized problem is quite involved. Even when convergence to the radial solution takes place (stability), the convergence is far from being monotone, with sustained oscillations present at all time.
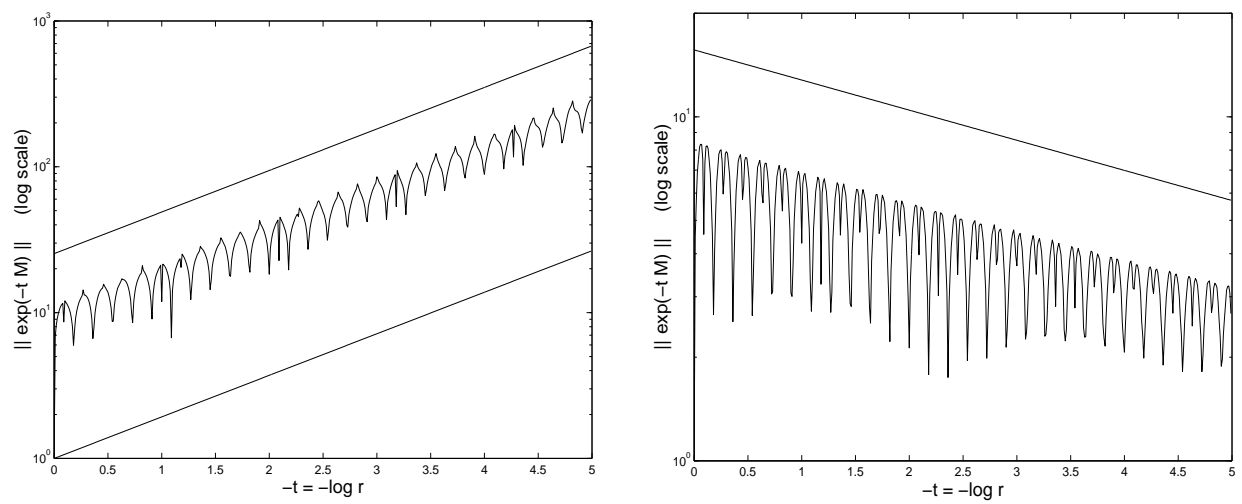

FiguRE 4.3. Behavior of $\|\exp (-t \mathbb{M})\|$ against $e^{-t \alpha(\mathbb{M})}$ and $\kappa(\mathbb{V}) e^{-t \alpha(\mathbb{M})}$ (straight lines) for $t$ increasingly negative for the problem of Figure 4.2; left: unstable two dimensional case, right: stable three dimensional case. 
We now turn to the study of the influence of the three parameters at hand, i.e., the wall friction, the internal friction and the opening angle on the stability of the linearized problem (4.1).
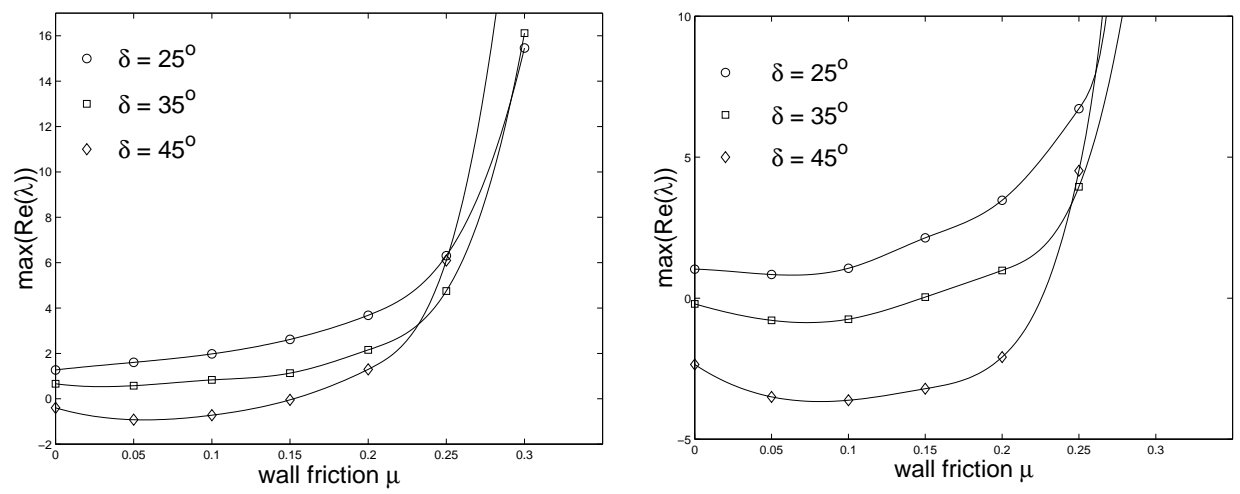

FigURE 4.4. Influence of the wall friction on the stability, for three values of the angle of internal friction $\delta=25^{\circ}, 35^{\circ}, 45^{\circ}$. Left: two dimensional case; right: three dimensional case.

Figure 4.4 illustrates two facts. First, when it is small, the wall friction has a stabilizing effect; however, for larger values, this effect becomes destabilizing. Second, for given material parameters, the two dimensional case is quite a lot "less stable" than its three dimensional counterpart.

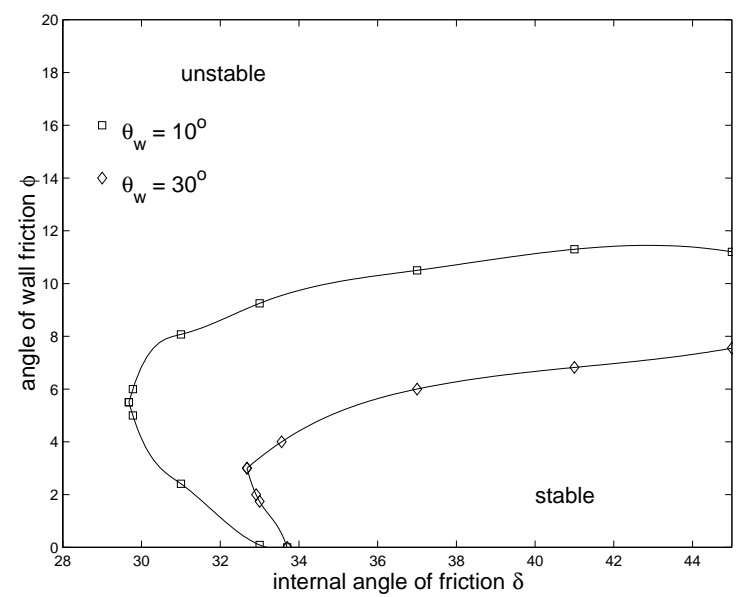

FIGURE 4.5. Dependency of the stability domain on the opening angle in the three dimensional case. The domains of stability correspond to the "interior" of the curves.

Finally, we consider the effect of the opening angle on the stability properties of the flow. Results are presented for the three dimensional case with opening angles of $10^{\circ}$ and $30^{\circ}$. Figure 4.5 illustrates the range of values of the angle internal friction $\delta$ and of the angle of wall friction $\phi$, defined by $\mu=\tan \phi$, corresponding to stability. 
It is observed that the domain of stability considerably shrinks as the opening angle increases.

\section{REFERENCES}

[1] G.C. BARKer, Computer simulations of granular materials, in Granular matter: an interdisciplinary approach, A. Metha (Ed.), Springer-Verlag, 1994, pp. 35-83.

[2] G.W. BaXter and R.P. Behringer, Cellular automata models for the flow of granular materials, Physica D, 51 (1991), pp. 465-471.

[3] R.P. Behringer And G.W. BaXter, Pattern formation and complexity in granular flows, in Granular matter: an interdisciplinary approach, A. Metha (Ed.), Springer-Verlag, 1994, pp. 85-119.

[4] C. Canuto, M.Y. Hussaini, A. Quarteroni and T.A. Zang, Spectral methods in Fluid Dynamics, Springer Verlag, 1988.

[5] P.A. Gremaud, Simulation of pattern formation in grain flows, Appl. Math. Comp., 84 (1997), pp. 145-162.

[6] P.A. Gremaud, D.G. Schaeffer and M. Shearer, Numerical determination of flow corrective inserts for granular materials in conical hoppers, submitted for publication in Int. J. Nonlinear Mechanics, Preprint CRSC-TR98-34, Center for Research in Scientific Computation, North Carolina State University, Raleigh, NC 27695-8205.

[7] A. JEnike, Gravity flow of bulk solids, Bulletin No. 108, Utah Eng. Expt. Station, University of Utah,Salt Lake City.

[8] A.T. Royal, Private Communication, Jenike \& Johnanson, Inc..

[9] J. R. Johanson, Stress and velocity fields in the gravity flow of bulk solids, J. Appl. Mech., 31 (1964), pp. 499-506.

[10] J. R. JOHANSON, The use of flow-corrective inserts in bins, Transactions of the ASME (1966), pp. 224-230.

[11] K. R. KAzA, The mechanics of flowing granular materials, Ph.D. Thesis, University of Houston, Houston, TX (1982).

[12] S.B.M. Moreea And R.M. Nedderman, Exact Stress and Velocity Distributions in a Cohesionless Material Discharging from a Conical Hopper, Chem. Eng. Sc., 51 (1996), pp. 39313942 .

[13] R.M. Nedderman, Statics and Kinematics of Granular Materials, Cambridge University Press, 1992.

[14] E. B. Pitman, The stability of granular flow in converging hoppers, SIAM J. Appl. Math., 48 (1988), pp. 1033-1052.

[15] D. G. SCHAEFFER, Instability in the evolution equations describing incompressible granular flow, J. Diff. Eqs., 66 (1987), pp. 19-50.

[16] L.N. Trefethen, Pseudospectra of linear operators, SIAM Rev., 39 (1997), pp. 383-406.

[17] L.N. Trefethen and M.R. Trummer, An instability phenomenon in spectral methods, SIAM J. Numer. Anal., 24 (1987), pp. 1008-1023. 